\title{
Shape bifurcation of a pressurized ellipsoidal balloon
}

\author{
Yanan Geng ${ }^{\mathrm{a}}$, Jiexiong Huang ${ }^{\mathrm{b}}$, Yibin $\mathrm{Fu}^{\mathrm{a}, \mathrm{c}, *}$ \\ ${ }^{a}$ Department of Mechanics, Tianjin University, Tianjin 300072, China \\ ${ }^{b}$ School of Naval Architecture, Ocean $\mathcal{E}$ Civil Engineering, Shanghai Jiao Tong University, Shanghai \\ 200240, China \\ ${ }^{c}$ Department of Mathematics, Keele University, Staffordshire ST5 5BG, UK
}

\begin{abstract}
It is well-known that for most spherical and cylindrical rubber balloons the pressure versus volume curve associated with uniform inflation both has an $N$-shape, but their shape bifurcation has different characters: whereas a spherical balloon tends to bifurcate into a pear shape through localized thinning near one of the poles, a cylindrical balloon would always bulge out locally in a symmetric manner. To understand the connection between these two different bifurcation behaviors, we study in this paper the shape bifurcation of an ellipsoidal balloon which becomes a spherical balloon when the three axes are identical, and approximates a cylindrical balloon when one axis is much larger than the other two axes. The ellipsoidal shape is obtained by rotating an ellipse about one of its axes, that gives rise to two possibilities: a rugby shape or a pumpkin shape. It is shown that for a rugby-shaped balloon, there exists a threshold axes ratio below which the slender ellipsoidal balloon behaves more like a tube and bifurcation into a pear shape becomes impossible, whereas for a pumpkin-shaped balloon bifurcation into a pear shape is always possible.
\end{abstract}

Keywords: Nonlinear elasticity, balloons, bifurcation, localization

\section{Introduction}

Inflating a rubber balloon is a classical problem in continuum mechanics. As a boundary-value problem it provided the first testing ground in the early development of the nonlinear elasticity theory (??), and as a bifurcation problem it is one of the first problems solved exactly using the incremental theory of nonlinear elasticity (??????). Turning-point instability, localized bulging, and bifurcation into a pear shape are phenomena commonly associated with pressurized balloons, and interest in these phenomena is still going strong because of a wide range of applications and because they also serve as a fertile ground for nonlinear analysis. More recent studies on dielectric spherical balloons

\footnotetext{
* Corresponding author at: Department of Mathematics, Keele University, Staffordshire ST5 5BG, UK Email address: y.fu@keele.ac.uk (Yibin Fu)
} 


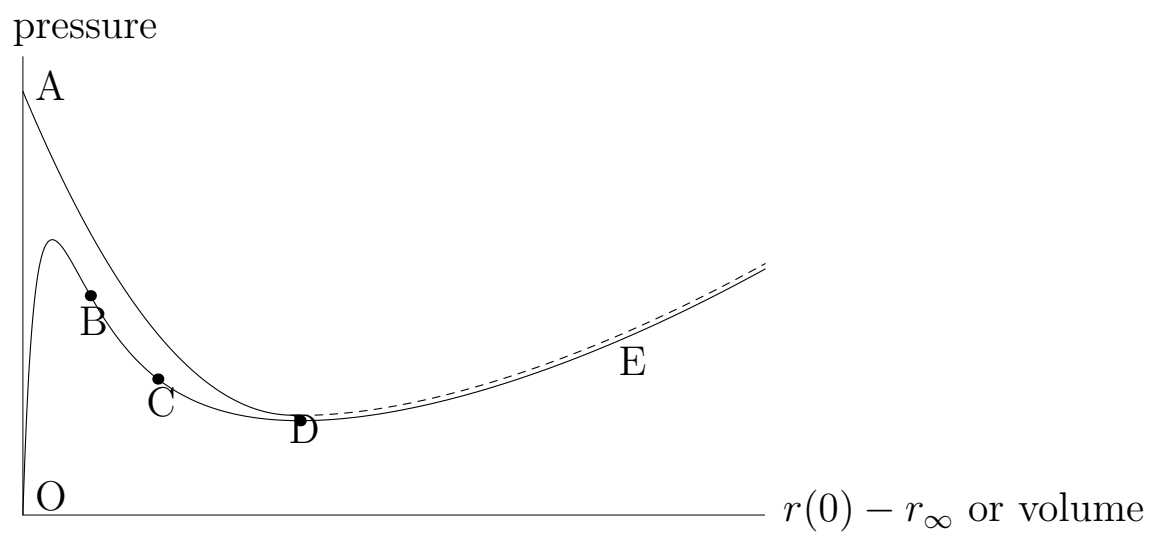

Figure 1: Typical bifurcation diagrams for a perfect tube (line AD) and a tube with imperfections (line OBCDE); in both cases the horizontal axis is $r(0)-r_{\infty}$ with $r(0)$ and $r_{\infty}$ denoting the radius at the center of the bulge and at infinity, respectively. Inflation of a spherical or ellipsoidal balloon follows a curve similar to OBCDE and points $\mathrm{B}$ and $\mathrm{C}$ would be where bifurcation into a pear shape occurs. In the latter interpretation the horizontal axis denotes the internal volume.

under the combined action of inflation and electric actuation (??), on fiber-reinforced hyperelastic tubes subject to additional swelling (?), and on balloons with viscoelastic and chemorheological effects (??) have provided renewed impetus into this research area. In particular, it was shown in ? that bifurcation into a pear shape is expected to be the norm when electric effects are taken into account; this is in contrast with the purely mechanical case in which such a bifurcation seems to be an exception in the sense that among the most commonly used rubber models only the Ogden material model allows such a shape bifurcation.

The present study is concerned with bifurcation into a pear shape of an ellipsoidal balloon, but it may also be viewed as a natural extension of our recent series of studies on localized bulging in cylindrical balloons. One of our objectives is to highlight the similarities and differences between the bifurcation behavior of the two types of membrane structures. For an inflated membrane tube, the most prominent feature is the formation of a localized bulge. When the tube has no inhomogeneities and is infinitely long (referred to as the perfect case), the appearance of a localized bulge is a subcritical bifurcation phenomenon (?), and the post-bifurcation behavior typically has the form of the upper curve shown in Fig. 1, where point $\mathrm{A}$ is the bifurcation point and point $\mathrm{D}$ corresponds to the stage when the bulge has grown to a maximum radius and further inflation will not change either the bulge amplitude or the pressure; instead the bulge will propagate axially in both directions. This bifurcation diagram can be "unfolded" by imperfections that may take the form of end constraints, material or geometrical inhomogeneities, or slow radius variation in the axial direction. In particular, for a finite tube with end constraints, the inflation behavior would take the form of the lower curve in Fig.1, and the longer the tube is, the closer the curve will be to the upper curve corresponding to the perfect case. It is of great interest to understand how much the initiation pressure is reduced in the presence 


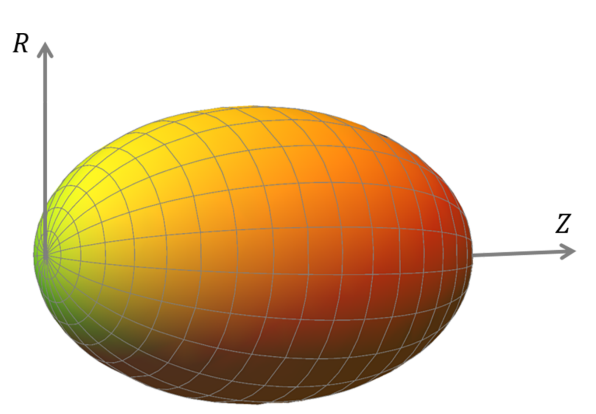

(a)

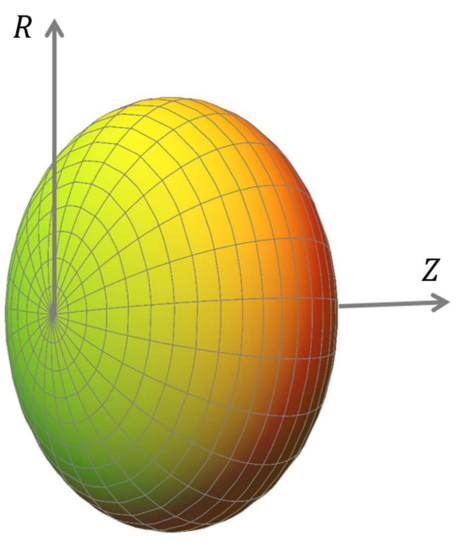

(b)

Figure 2: Two shapes of the ellipsoidal balloon.

of imperfections (??). Also, for a long but finite tube, the appearance of a localized bulge will not be as "clean" as in the perfect case, and the propagation of the bulge will soon terminate when the entire tube has reached a (almost) constant radius. If inflation continues, the tube will stiffen and it will follow the curve DE in Fig.1. When the tube is short enough, localized bulging may not be observable at all, and the inflation process is more like that associated with a spherical balloon. However, for the latter situation, bifurcation into a pear shape will occur when the pressure lies in a sub interval between points $\mathrm{B}$ and $\mathrm{C}$ in Fig.1, but such bifurcations are not known for cylindrical tubes.

In order to resolve the above difference in the bifurcation behavior, we consider the inflation of an ellipsoidal balloon the surface of which is formed by revolving an ellipse in the $Z R$-plane around the $Z$-axis; see Fig.2a for the case when $a / b>1$ and Fig.2b for the case when $a / b<1$, where $a$ and $b$ are the semi-axes in the $Z$-and $R$-directions, respectively. We note that the case $b=a$ would correspond to the spherical balloon case, whereas the case $b \ll a$ can be used to approximate a cylindrical balloon whose radius varies slowly in the axial direction. The inflation of an incomplete ellipsoidal balloon, that is an ellipsoidal balloon that has been truncated at some value of $Z$, has previously been analyzed by ?.

The rest of this paper is divided into four sections as follows. After formulating the inflation problem in the next section, we determine all possible axially symmetric solutions, either symmetric or non-symmetric about the middle vertical plane, of a pressurized ellipsoidal balloon by adapting the procedures used in ? and ?. Hereafter these two types of axially symmetric solutions will be referred to as the primary and bifurcated solutions, respectively. In the case of an initially spherical balloon, the primary solution would correspond to a spherical configuration with a larger radius. We examine both rugby-shaped and pumpkin-shaped balloons, and clarify the associated bifurcation behavior. The paper is concluded in the final section with a summary and further discussion of the similarities and differences in the inflation of cylindrical and spherical balloons. 


\section{Governing equations}

We consider an ellipsoidal balloon that is described by

$$
R(\theta)=b \sin \theta, \quad Z(\theta)=a(1-\cos \theta), \quad 0 \leq \theta \leq \pi,
$$

in terms of cylindrical polar coordinates $(R, Z)$ in its undeformed configuration (note that $\theta$ is not part of the polar coordinates but a parameter), where $a$ and $b$ are the semi-axes of the ellipse whose rotation about the $Z$-axis forms the ellipsoidal balloon. There now exist two possibilities: $a>b$ and $a<b$, as sketched in Figs 3 and 4 , respectively. In the first case, the two identical axes are smaller than the third axis and the balloon is of a rugby shape, see Fig.2a. In the second case, the two identical axes are larger than the third axis and the balloon is of a pumpkin shape, see Fig.2b. To facilitate interpretation of numerical results, we use $\max \{a, b\}$ as the length unit in both cases. Thus, for a rugbyshaped balloon, we take $a=1, b<1$, whereas for a pumpkin-shaped balloon we take $b=1, a<1$.
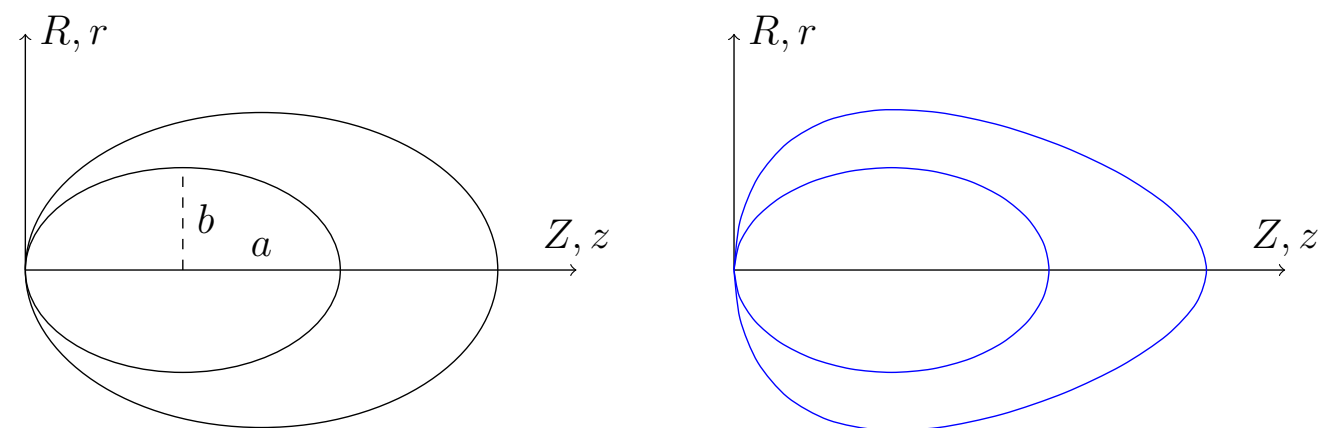

Figure 3: Axially symmetric (about the $Z$-axis) deformation of a rugby-shaped balloon. Outer curve on the left: primary deformed configuration that is symmetric about the middle vertical plane; outer curve on the right: bifurcated configuration that is pear-shaped. In both figures the inner curve is the undeformed configuration.

We focus on axisymmetric deformations described by

$$
r=r(\theta), \quad z=z(\theta),
$$

where $r$ and $z$ are cylindrical polar coordinates in the deformed configuration. Denote by $d S$ and $d s$ the arclengths measured from $\theta=0$ in the reference and current configurations, respectively. We then have

$$
d S=\sqrt{(d R)^{2}+(d Z)^{2}}=\chi(\theta) d \theta, \quad d s=\sqrt{\left(\frac{d r}{d \theta}\right)^{2}+\left(\frac{d z}{d \theta}\right)^{2}} d \theta
$$

where the function $\chi(\theta)$ is defined by

$$
\chi(\theta)=\sqrt{a^{2} \sin ^{2} \theta+b^{2} \cos ^{2} \theta} .
$$



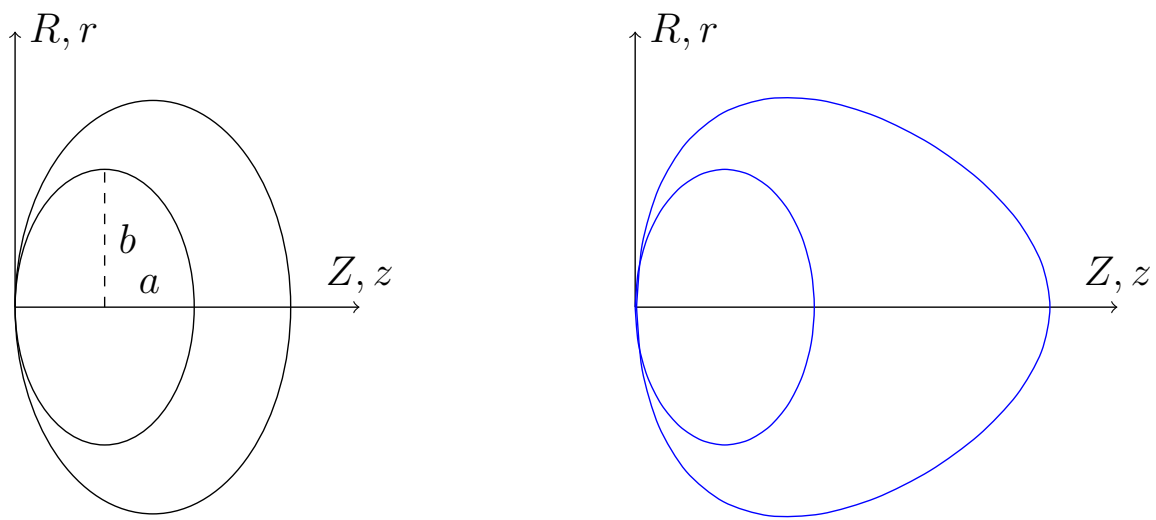

Figure 4: Axially symmetric (about the $Z$-axis) deformation of a pumpkin-shaped balloon. The curves have the same meanings as in Figure 3.

Since the deformation is axially symmetric, the principal directions of stretch coincide with the lines of latitude, the meridian and the normal to the deformed surface. Thus, the principal stretches are given by

$$
\lambda_{1}=\frac{r}{R}, \quad \lambda_{2}=\frac{d s}{d S}=\frac{\mu_{2}}{\chi(\theta)} \equiv \frac{1}{\chi(\theta)} \sqrt{\left(\frac{d r}{d \theta}\right)^{2}+\left(\frac{d z}{d \theta}\right)^{2}}, \quad \lambda_{3}=\frac{h}{H},
$$

where $H$ and $h$ are the undeformed and deformed thicknesses, respectively, and the middle expression serves to define the function $\mu_{2}$.

By considering equilibrium of an infinitesimal volume element in the 1- and 2- directions, respectively, we obtain

$$
\begin{gathered}
r\left(h \sigma_{2}\right)^{\prime}+h r^{\prime}\left(\sigma_{2}-\sigma_{1}\right)=0, \\
\frac{z^{\prime} \sigma_{1}}{r \lambda_{2}}+\frac{\sigma_{2}\left(r^{\prime} z^{\prime \prime}-z^{\prime} r^{\prime \prime}\right)}{\lambda_{2}^{3}}=\lambda_{1} \lambda_{2} \bar{P},
\end{gathered}
$$

where $\sigma_{1}$ and $\sigma_{2}$ denote the principal Cauchy stresses, a prime denotes differentiation with respect to the arclength $S$, and $\bar{P}$ is the actual pressure $P$ scaled by $\mu \mathrm{H}$. The two equilibrium equations have one integral, which corresponds to the constancy (zero in this case) of the resultant force at any cross-section, and is given by

$$
z^{\prime}=\frac{\bar{P} r \lambda_{1} \lambda_{2}^{2}}{2 \sigma_{2}} .
$$

In particular, at any cross section where $r^{\prime}=0$, we have

$$
1=\frac{\bar{P} R(\theta) \lambda_{1}^{2} \lambda_{2}}{2 \sigma_{2}}
$$

which can be used to express $\lambda_{2}$ in terms of $\lambda_{1}$ at that particular cross section.

The equilibrium equations may also be expressed in terms of the principal curvatures

$$
\kappa_{1}=\frac{\cos \phi}{r}, \quad \kappa_{2}=-\frac{d \phi}{d s}=\frac{(\cos \phi)^{\prime} d S}{\sin \phi \lambda_{2} d S}=\frac{r^{\prime} z^{\prime \prime}-z^{\prime} r^{\prime \prime}}{\lambda_{2}^{3}},
$$


where $\phi$ is the angle between the meridian and the $z$-axis. The equilibrium equations then take the alternative form

$$
\left(h \sigma_{2} r\right)^{\prime}=\left(h \sigma_{1}\right) r^{\prime}, \quad \kappa_{1}\left(h \sigma_{1}\right)+\kappa_{2}\left(h \sigma_{2}\right)=H \bar{P}
$$

see, e.g., ?. With the use of the relations $r^{\prime}=\lambda_{2} \sin \phi, \quad z^{\prime}=\lambda_{2} \cos \phi$, the equilibrium equations (5) and (6) can be converted into the following system of first-order ordinary differential equations:

$$
\begin{aligned}
\frac{d \lambda_{1}}{d \theta} & =\frac{\lambda_{2} \chi(\theta) \sin \phi-b \lambda_{1} \cos \theta}{b \sin \theta} \\
\frac{d \lambda_{2}}{d \theta} & =\frac{W_{1}-\lambda_{2} W_{12}}{b W_{22}} \cdot \frac{\chi(\theta) \sin \phi}{\sin \theta}-\frac{W_{2}-\lambda_{1} W_{12}}{W_{22}} \cdot \cot \theta \\
\frac{d \phi}{d \theta} & =\frac{W_{1}}{W_{2}} \frac{\chi(\theta) \cos \phi}{b \sin \theta}-\frac{\bar{P} \lambda_{1} \lambda_{2} \chi(\theta)}{W_{2}}
\end{aligned}
$$

where $W_{1}=\partial W / \partial \lambda_{1}, W_{12}=\partial^{2} W / \partial \lambda_{1} \partial \lambda_{2}$ etc, and $W\left(\lambda_{1}, \lambda_{2}\right)=\tilde{W}\left(\lambda_{1}, \lambda_{2}, \lambda_{1}^{-1} \lambda_{2}^{-1}\right)$, $\tilde{W}$ being the three-dimensional strain-energy function (measured per unit volume in the undeformed configuration). In writing down the above equations, use has also been made of the constitutive relations $\sigma_{1}=\lambda_{1} W_{1}, \sigma_{2}=\lambda_{2} W_{2}$.

In our example calculations, we shall adopt the Ogden material model given by

$$
\begin{gathered}
W=\sum_{r=1}^{3} \tilde{\mu}_{r}\left(\lambda_{1}^{\alpha_{r}}+\lambda_{2}^{\alpha_{r}}+\lambda_{3}^{\alpha_{r}}-3\right) / \alpha_{r} \\
\alpha_{1}=1.3, \alpha_{2}=5.0, \alpha_{3}=-2.0, \tilde{\mu}_{1}=1.491, \tilde{\mu}_{2}=0.003, \tilde{\mu}_{3}=-0.024
\end{gathered}
$$

where $W$ has been scaled by the ground state shear modulus; see ?.

\section{The primary and bifurcated inflation solutions}

The system of equations (10) are now integrated numerically to obtain all fully nonlinear solutions that may exist. We use a similar procedure to the one used in ? and ?. It is first noted that the system of equations (10) has a removable singularity at the two poles $\theta=0, \pi$. Thus to avoid evaluation at the poles, we integrate from $\theta=\delta$ to $\theta=\pi-\delta$ instead, where $\delta$ is a sufficiently small constant. We then need sufficiently accurate estimates for the values of $\lambda_{1}, \lambda_{2}$ and $\phi$ at $\theta=\delta$. To simplify notation, from now on a prime will denote differentiation with respect to $\theta$.

Because of the axi-symmetry at the poles, we first have

$$
\lambda_{1}(0)=\lambda_{2}(0), \quad \lambda_{1}(\pi)=\lambda_{2}(\pi), \quad \phi(0)=\frac{\pi}{2}, \quad \phi(\pi)=-\frac{\pi}{2},
$$

and

$$
\lambda_{1}^{\prime}(0)=\lambda_{2}^{\prime}(0)=\phi^{\prime \prime}(0)=0
$$


On differentiating $(10)_{1,2}$ with respect to $\theta$ and then evaluating at the two poles, we obtain two linear equations for $\lambda_{1}^{\prime \prime}$ and $\lambda_{2}^{\prime \prime}$, the solution of which then gives

$$
\begin{aligned}
& \lambda_{1}^{\prime \prime}=\frac{\left(3 \lambda_{1} W_{11}-\lambda_{1} W_{12}+W_{1}\right)\left(a^{2}-b^{2} \phi^{2}\right)}{8 b^{2} W_{11}}, \quad \theta=0, \pi, \\
& \lambda_{2}^{\prime \prime}=\frac{\left(\lambda_{1}\left(W_{11}-3 W_{12}\right)+3 W_{1}\right)\left(a^{2}-b^{2} \phi^{2}\right)}{8 b^{2} W_{11}}, \quad \theta=0, \pi .
\end{aligned}
$$

Expanding $(10)_{3}$ at the two poles and solving the resulting equations, we obtain, at $\theta=0, \pi$

$$
\begin{gathered}
\phi^{\prime}=-\frac{b \bar{P} \lambda_{1}^{2}}{2 W_{2}} . \\
\phi^{\prime \prime \prime}=\frac{\lambda_{1} \bar{P}}{64 b W_{1}^{4} W_{11}}\left\{6 \lambda_{1}^{2} W_{1}^{2}\left(2 a^{2} W_{11}^{2}-2 a^{2} W_{12}^{2}+b^{4} \lambda_{1}^{2} \bar{P}^{2}\right)\right. \\
-4 \lambda_{1} W_{1}^{3}\left(W_{11}\left(9 a^{2}-8 b^{2}\right)-9 a^{2} W_{12}\right)-24 a^{2} W_{1}^{4} \\
\left.+3 b^{4} \lambda_{1}^{6} \bar{P}^{2}\left(W_{12}^{2}-W_{11}^{2}\right)+b^{4} \lambda_{1}^{5} \bar{P}^{2} W_{1}\left(W_{11}-9 W_{12}\right)\right\},
\end{gathered}
$$

where the right hand side is evaluated at the respective pole. It then follows that

$$
\begin{aligned}
\lambda_{1}(\delta) & =\lambda_{1}(0)+\frac{1}{2} \lambda_{1}^{\prime \prime}(0) \delta^{2}+O\left(\delta^{4}\right), \\
\lambda_{2}(\delta) & =\lambda_{1}(0)+\frac{1}{2} \lambda_{2}^{\prime \prime}(0) \delta^{2}+O\left(\delta^{4}\right), \\
\phi(\delta) & =\frac{\pi}{2}+\phi^{\prime}(0) \delta+\frac{1}{6} \phi^{\prime \prime \prime}(0) \delta^{3}+O\left(\delta^{5}\right),
\end{aligned}
$$

and near the pole $\theta=\pi$, we have

$$
\begin{gathered}
\frac{\pi}{2}+\phi(\pi-\delta)+\phi^{\prime}(\pi-\delta) \delta=O\left(\delta^{3}\right), \\
\lambda_{1}(\pi-\delta)-\lambda_{2}(\pi-\delta)+\delta\left\{\lambda_{1}^{\prime}(\pi-\delta)-\lambda_{2}^{\prime}(\pi-\delta)\right\}=O\left(\delta^{2}\right) .
\end{gathered}
$$

These expressions reduce to their counterparts given by Fu and Xie (2014) in the special case when $a=b$.

Thus, the initial data $\lambda_{1}(\delta), \lambda_{1}(\delta)$, and $\phi(\delta)$ can be expressed in terms of a single unknown parameter, $\lambda_{1}(0)$ say. The system of ODEs (10) can then be solved using a shooting method. We iterate on $\lambda_{1}(0)$ so that the boundary/target condition (19) or (20) is satisfied. By varying $\lambda_{1}(0)$ over the entire range $(1, \infty)$, this approach enables us to find all possible solutions at any given pressure, including both primary and bifurcated solutions when they exist. For instance, in the problem of an inflated spherical balloon, we could find three primary solutions and other two pear-shaped solutions when set the pressure at the interval where bifurcation is possible. This procedure is straightforward; the only numerical difficulty is that off-target solutions tend to blow up when they reach the other pole. Numerical experimentation shows that any valid solution tends to reside near solutions that blow up when the end $\theta=\pi-\delta$ is reached. Thus, we usually first find 
a value of $\lambda_{1}(0)$ across which the expression in the target condition (19) or (20) changes from being finite to being infinite, and then refine the increment in $\lambda_{1}(0)$ to locate a value across which the expression in the target condition changes sign. This strategy works well except when $b / a$ becomes too small.

Our numerical code is also validated by considering only the primary solutions, that is those that are symmetric about the middle vertical plane $\theta=\pi / 2$. In this case we only need to integrate to $\theta=\pi / 2$ where the target condition is $\phi=0$; off-target solutions usually do not blow up when the end $\theta=\pi / 2$ is reached. Alternatively, we may consider the interval $\pi / 2<\theta<\pi-\delta$. At $\theta=\pi / 2, \phi=0$ and $\lambda_{2}$ can be expressed in terms of $\lambda_{1}$ by solving (8). We may then integrate the system (10) from $\theta=\pi / 2$ to $\theta=\pi-\delta$, and iterate on $\lambda_{1}(\pi / 2)$ to satisfy the target condition (19) or (20). After some experimentation, we find it sufficient to choose $\delta=0.01$; choosing a smaller value of $\delta$ only improves the accuracy of the final $\lambda_{1}(0)$ in a negligible manner (typically digits after the fourth decimal place).

We shall present our numerical results for a rugby-shaped balloon and a pumpkinshaped balloon separately. In both cases, we take the semi-major axis to be the length unit so that we have $a=1$ and $0<b<1$ for the rugby-shaped balloon, and $b=1$ and $0<a<1$ for the pumpkin-shaped balloon. For both cases considered, we find that the pressure versus volume curve for the primary deformation is of the form OBCDE in Fig.1. The primary configuration may bifurcate into a pear shape when point $\mathrm{B}$ on the curve is reached, and the bifurcated solution disappears when point $\mathrm{C}$ further down the curve is reached. However, there are some major differences between the two cases which are discussed below.

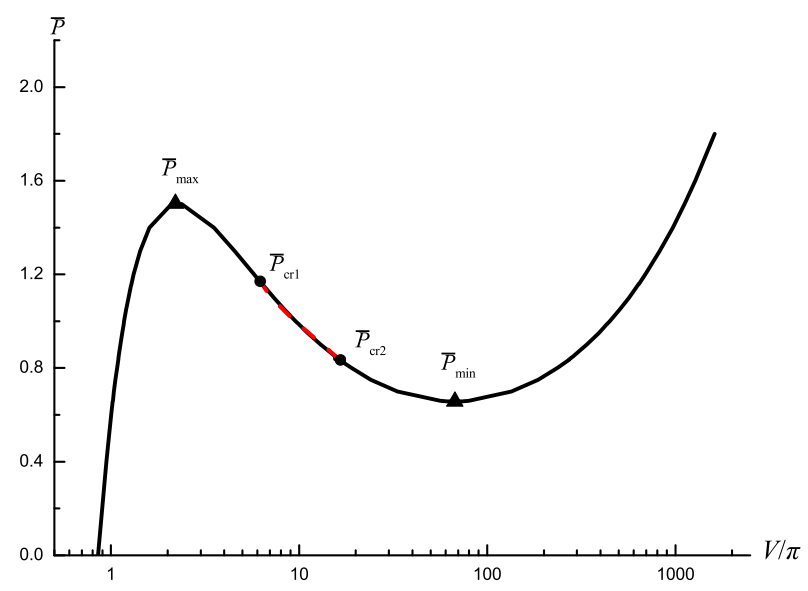

Figure 5: Variation of pressure with respect to volume when $a=1, b=0.8$. The interval $\left(\bar{P}_{c r 1}, \bar{P}_{c r 2}\right)$ is where bifurcation takes place. The pressure for the primary and bifurcated solutions are inextinguishable in this figure; see Fig. 6 for a blow-up. 


\subsection{Inflation of a rugby-shaped balloon}

This corresponds to the case shown in Fig. 3. As will become clear shortly, bifurcated solutions may or may not exist depending on the slenderness of the balloon. Results corresponding to a representative case with $a=1, b=0.8$ when bifurcation into a pear shape is possible are displayed in Fig. 5 and Fig. 6. In this case, we have

$$
\bar{P}_{\max }=1.502, \quad \bar{P}_{\text {cr } 1}=1.1598, \quad \bar{P}_{\text {cr2 }}=0.8369, \quad \bar{P}_{\text {min }}=0.658,
$$

where $\bar{P}_{\max }$ and $\bar{P}_{\text {min }}$ denote the pressure maximum and minimum, respectively, and $\bar{P}_{\text {cr1 }}$ and $\bar{P}_{\text {cr2 }}$ the two ends of the interval where bifurcated solutions exist.

We note that although the pressure versus volume curves are almost indistinguishable for the primary and bifurcated solutions, the distribution of the two principal stretches associated with the two solutions are very different. The curve for the primary solution is symmetric about the middle vertical plane $\theta=\pi / 2$, while the one for the bifurcated solution has a minimum value at the pole $\theta=0$. One more thing we should notice is that when $\bar{P}=1$, the shapes of the primary and bifurcated solutions are both like a spherical balloon, so $\lambda_{1}$ and $\lambda_{2}$ have almost the same distribution over the membrane for both kinds of shapes. But for other pressure, near $\bar{P}_{\max }$ and $\bar{P}_{\text {min }}$ for example, the deformed shape corresponding to the second primary solution is ellipsoidal balloon.
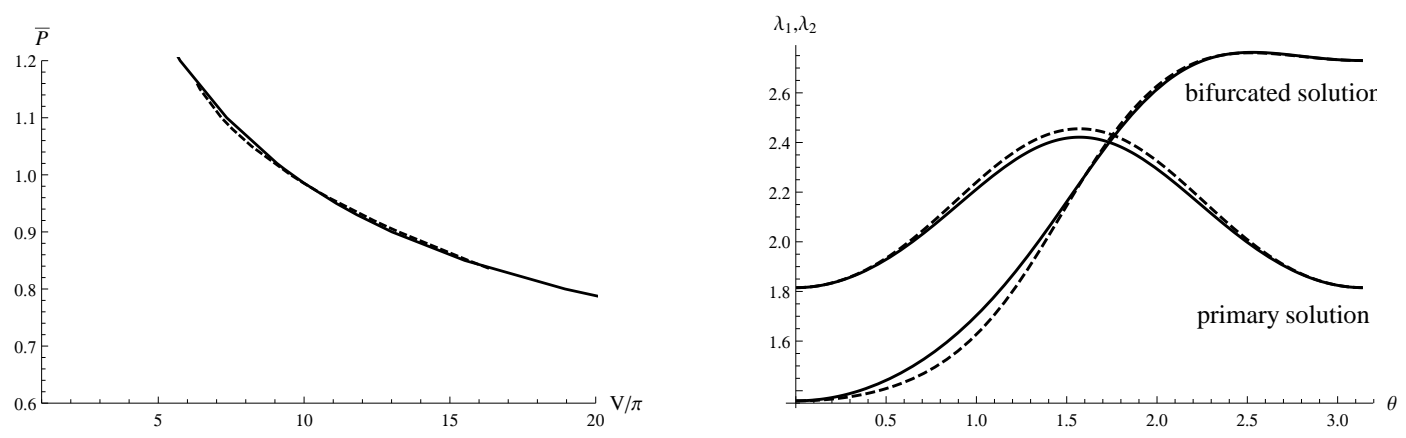

Figure 6: Left: Variation of pressure with respect to volume on the descending branch with the dashed and solid lines representing the bifurcated and primary solutions, respectively. Right: variations of $\lambda_{1}$ (solid lines) and $\lambda_{2}$ (dashed lines) with respect to $\theta$ when $a=1, b=0.8$ and $\bar{P}=1$.

The total energy, scaled by $2 \pi H \mu$, can be computed with the aid of the expression

$$
\bar{E}=\int_{0}^{\pi}\left[W\left(\lambda_{1}, \lambda_{2}\right) b \sin \theta \chi(\theta)-\frac{1}{2} \bar{P} r^{2} z^{\prime}\right] \mathrm{d} \theta,
$$

and we shall use $\bar{E}_{u}$ and $\bar{E}_{b}$ to denote its values for the primary and bifurcated solutions, respectively. Fig. 7 shows their difference in the pressure interval $\left(\bar{P}_{\mathrm{cr} 2}, \bar{P}_{\mathrm{cr} 1}\right)$, and it is seen that the pear-shaped configuration has lower energy whenever it can exist; this is qualitatively similar to the case of a spherical balloon.

We next examine how the above behavior is changed when $b$ becomes increasingly smaller and smaller. The values of $\bar{P}_{c r 1}$ and $\bar{P}_{c r 2}$ for a selection of values of $b$ are presented 


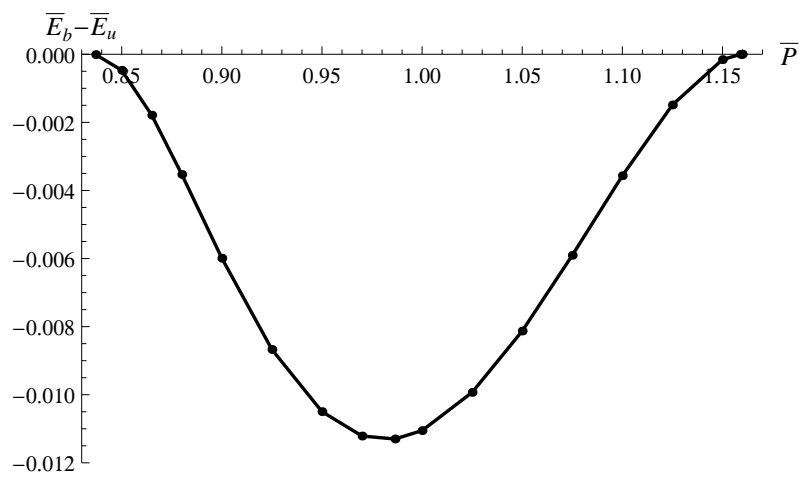

Figure 7: Difference in the total energy between bifurcated and primary solution.

in Table 1. It is seen immediately that as $b$ is decreased, the interval $\left(\bar{P}_{c r 1}, \bar{P}_{c r 2}\right)$ where bifurcation can take place becomes smaller and smaller, vanishing completely when $b$ reaches a threshold value $b_{\text {cr }}$ approximately equal to 0.626. Thus, we may conclude that when $b \geq b_{c r}$, apart from three primary solutions, there also exist two other bifurcated solutions, which are mirror images of each other. However, when $b<b_{c r}$, the bifurcated solutions will not appear any more. This is consistent with the anticipation that when the balloon is slender enough, it should be more like a cylindrical tube and for the latter bifurcation into a pear shape is known to be impossible.

\begin{tabular}{|c|c|c|c|c|}
\hline$b$ & $\bar{P}_{\max }$ & $\bar{P}_{c r 1}$ & $\bar{P}_{c r 2}$ & $\bar{P}_{\min }$ \\
\hline 0.8 & 1.5026 & 1.15982 & 0.8369 & 0.65735 \\
\hline 0.7 & 1.6312 & 1.1356 & 0.89564 & 0.71995 \\
\hline 0.65 & 1.7064 & 1.0904 & 0.9468 & 0.75817 \\
\hline 0.62614 & 1.7453 & 1.01922 & 1.0182 & 0.77855 \\
\hline
\end{tabular}

Table 2: Values of $\lambda_{1}(\delta)$ when $b=0.25$

\begin{tabular}{ccccc}
\hline & $\bar{P}$ & $\lambda_{1}^{(1)}(\delta)$ & $\lambda_{1}^{(2)}(\delta)$ & $\lambda_{1}^{(3)}(\delta)$ \\
\hline & 0.1 & 1.0005 & & \\
$\bar{P}_{\text {min }}$ & 1.5 & 1.0086 & & \\
& 2 & 1.01195 & 1.01196 & 2.00000 \\
& 3.35 & 1.02277 & 1.02278 & 5.34096 \\
$\bar{P}_{\max }$ & 3.361 & 1.02288 & 1.022883 & 5.35755 \\
& 3.5 & & & 5.56199 \\
& 3.8 & & & 5.97576 \\
\hline
\end{tabular}

To understand more precisely how a slender ellipsoidal balloon behaves like a cylindrical balloon, four typical pressure/volume curves, corresponding to $b=0.5,0.4,0.3,0.25$, 


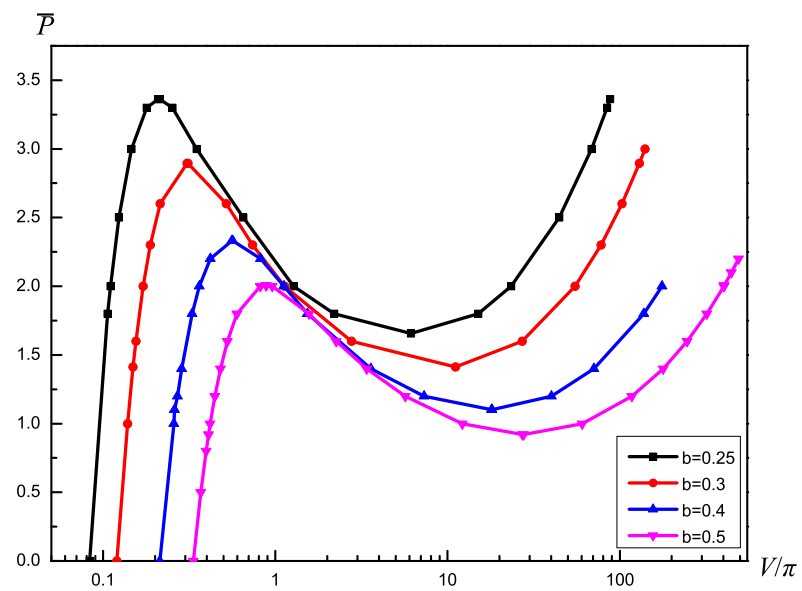

Figure 8: Variation of pressure with respect to volume for increasingly smaller and smaller values of $b$.

respectively, are shown in Fig. 8. Although the three segments/branches in each curve are well separated due to the use of logarithmic scale, the values of $\lambda_{1}(\delta)$ for the first two primary solutions are in fact very close, as can be seen in Table 2 where these values, denoted by $\lambda_{1}^{(1)}(\delta)$ and $\lambda_{1}^{(2)}(\delta)$, are listed for the case when $b=0.25$ (the $\lambda_{1}^{(3)}(\delta)$ in this table corresponds to the third solution). The closeness of these two values are indicative of the fact that the solution corresponding to $\lambda_{1}^{(2)}(\delta)$ is a localized bulging solution that bifurcated from the first solution corresponding to $\lambda_{1}^{(1)}(\delta)$. In the case of an infinitely long cylindrical balloon, the first solution would be the uniformly inflated solution and the values of $\lambda_{1}^{(1)}$ and $\lambda_{1}^{(2)}$ at the ends, which are now at infinity, would be identical. To lend more support to this claim, we also note that in the perfect case, a near-critical localized bulging solution would take the form $\lambda_{2}=c_{1}+c_{2} \operatorname{sech}^{2}\left(c_{3}(Z-a)\right)$ for some constants $c_{1}, c_{2}$ and $c_{3}$; see ?. In Fig. 9, we have shown the profile of $\lambda_{2}$ corresponding to $\bar{P}=3.3, b=0.25$ on the descending branch against the approximate expression

$$
\lambda_{2}=1+0.36 \operatorname{sech}^{2}(2.55(Z-a)) .
$$

It is seen that the actual variation of $\lambda_{2}$ is well represented by the solitary-wave type solution (22) except in a small region adjacent to each of the two poles, the discrepancy near the two poles representing end/boundary effects. Fig. 10 displays the corresponding undeformed and deformed shapes of the balloon, which may be compared with the deformed shape of a cylindrical tube with plugged ends presented in ?.

It is also of interest to compare the initiation pressure $\bar{P}_{\max }=3.361$ for the case $b=0.25$ with its counterpart $P_{\text {cr }}$ corresponding to an infinitely long cylindrical tube of radius $b$. The latter value is given by

$$
P_{\text {cr }}=0.75 \frac{\mu H}{b} \text {. }
$$

See, e.g., ?. Recalling that $\bar{P}$ used in this paper is the actual pressure scaled by $\mu H$ and we have used $a$ to non-dimensionalize our length variables and parameters, the initiation 
value $\bar{P}_{\max }=3.361$ corresponds to an actual pressure value of

$$
3.361 \cdot \frac{\mu H}{a}=3.361 \cdot \frac{\mu H}{b} \cdot \frac{b}{a}=0.25 \times 3.361 \cdot \frac{\mu H}{b}=0.84 \frac{\mu H}{b} .
$$

Surprisingly, this seems to show that an ellipsoidal balloon is more bulging-resistant than a tubular balloon!

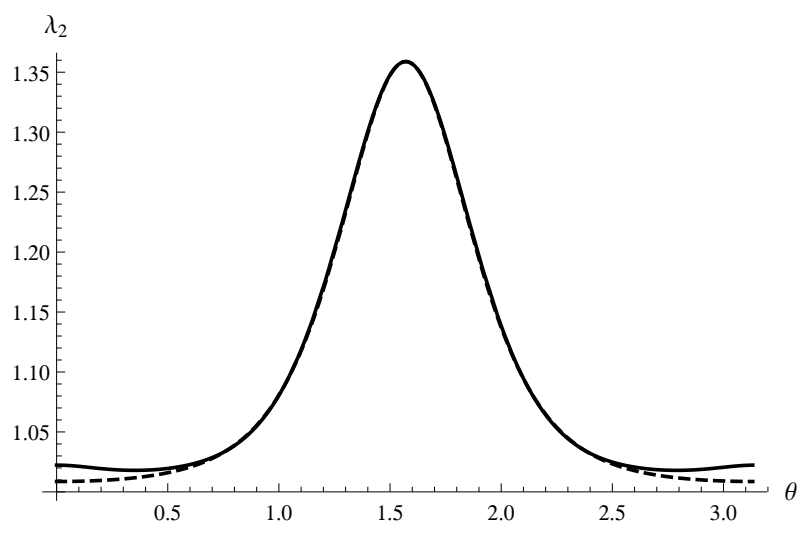

Figure 9: Profile of $\lambda_{2}$ corresponding to $a=1, b=0.25$ and $\bar{P}=3.3$ on the descending branch (solid line) compared with the solitary-wave type approximation (22) (dashed line).

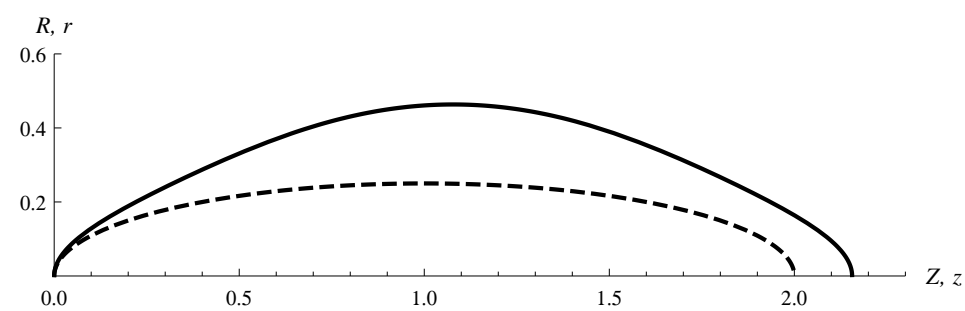

Figure 10: The undeformed (dashed line) and deformed shapes (solid line) of the ellipsoidal balloon when $a=1, b=0.25, \bar{P}=3.3$.

\subsection{Inflation of a pumpkin-shaped balloon}

In this case we use $b$ as the length unit so that after non-dimensionalisation $b$ becomes unity and $a$ varies between 0 to 1 .

Our first calculation is for the case when $a=0.8$, and it is found that there exists a sub-interval of pressure, $\left(\bar{P}_{\mathrm{cr} 2}, \bar{P}_{\mathrm{cr} 1}\right)$, in which bifurcated solutions as well as the primary solutions can exist. The associated pressure variations are shown in Fig. 11 and Fig. 12. In this case, we have

$$
\bar{P}_{\max }=1.39426, \quad \bar{P}_{\mathrm{cr} 1}=1.22890, \quad \bar{P}_{\mathrm{cr} 2}=0.86965, \quad \bar{P}_{\min }=0.60903 .
$$

Furthermore, by comparing their energies with the aid of (21), we again find that the 


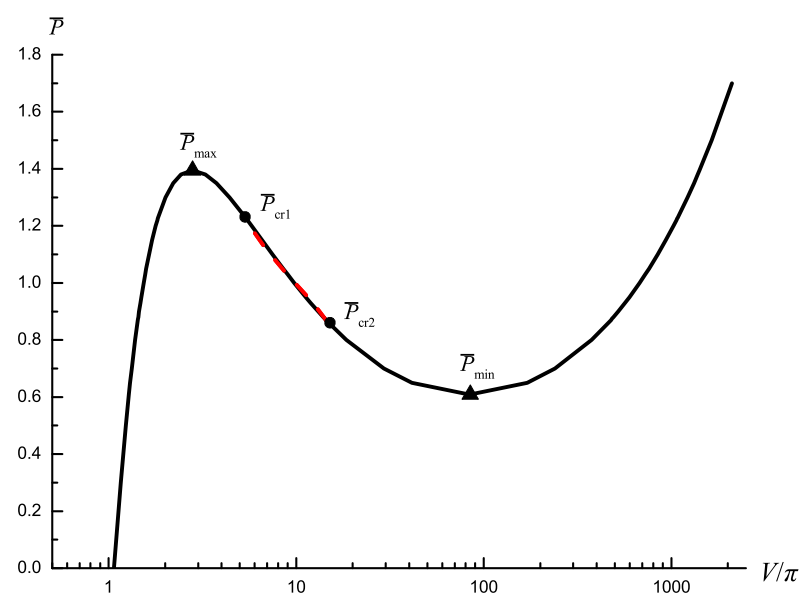

Figure 11: Variation of pressure with respect to volume when $b=1, a=0.8$ and the interval of $\bar{P}_{c r 1}$ and $\bar{P}_{c r 2}$ would be the range where bifurcation exists.
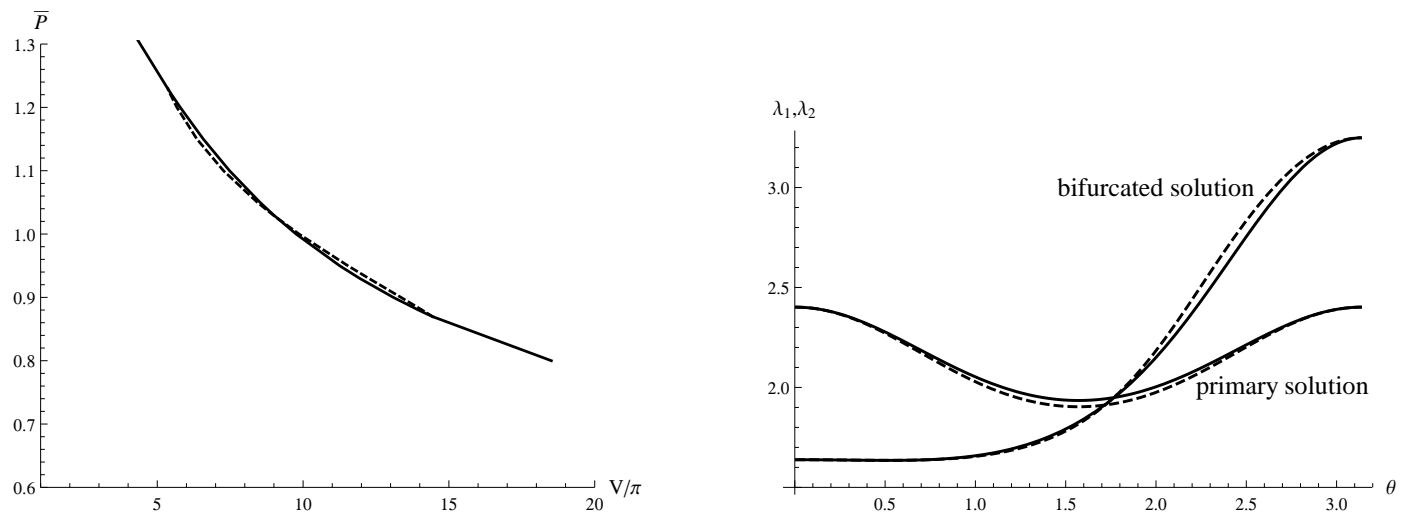

Figure 12: Left: Variation of pressure with respect to volume on the descending branch. Dashed line: bifurcated solution; solid line: primary solution. Right: Variations of $\lambda_{1}$ (solid lines) and $\lambda_{2}$ (dashed lines) with respect to $\theta$ when $b=1, a=0.8$ and $\bar{P}=1$.

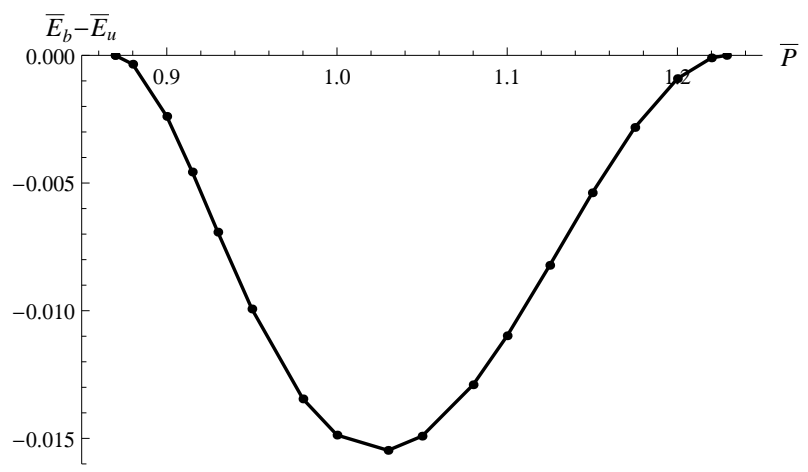

Figure 13: Difference in the total energy between bifurcated and primary solution.

bifurcated solutions have lower energies than the primary solutions, which is depicted in Fig. 13. 
We next examine what happens to the bifurcation interval when $a$ is decreased even further. It is found that the bifurcation interval $\left(\bar{P}_{c r 1}, \bar{P}_{c r 2}\right)$ persists no matter how small the semi-minor axis $a$ may be. For instance, for $a=10^{-6}$, we have

$$
\bar{P}_{\max }=1.8110, \quad \bar{P}_{\text {cr1 }}=1.6036, \quad \bar{P}_{\text {cr2 }}=1.3861, \quad \bar{P}_{\min }=0.8305 .
$$
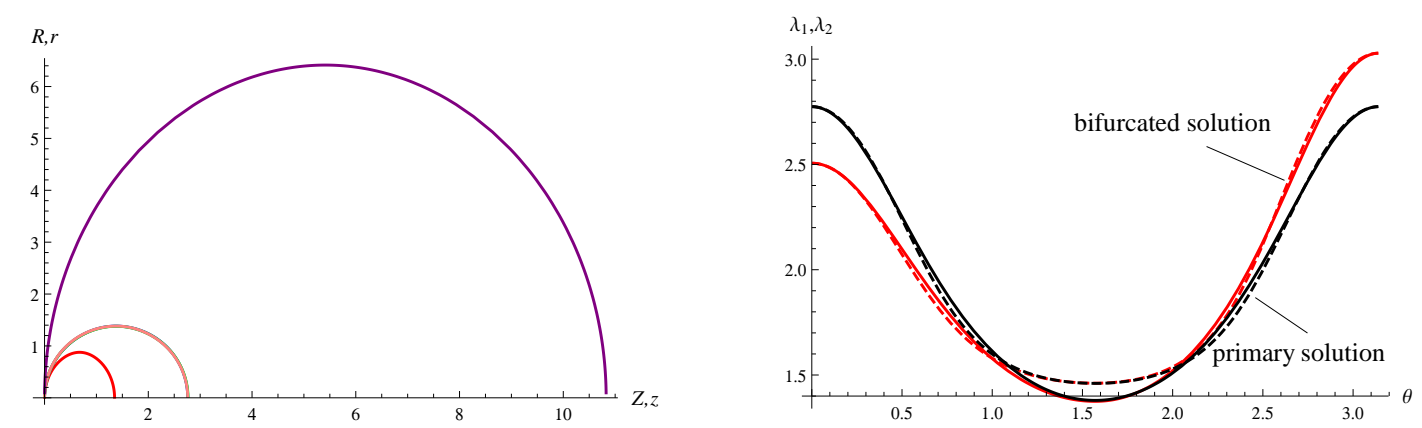

Figure 14: Left: Shapes of the three primary and the two bifurcated solutions. Right: Variations of $\lambda_{1}$ (solid lines) and $\lambda_{2}$ (dashed lines) with respect to $\theta$ when $b=1, a=10^{-6}$ and $\bar{P}=1.4$.

We also note that the change in the shape of the ellipsoidal balloon from its initial shape is quite significant, and the balloon approaches a spherical shape rapidly. For instance, when $\bar{P}=1.4$, at which value both bifurcated solution and primary solutions can exist, Fig. 14a shows the shapes of all the solutions that can exist: the smallest one is the shape of the first primary solution, the middle ones are the shape of the second primary and the two bifurcated solutions, which are almost inextinguishable, and the largest one is the shape of the third primary solution. The initial shape with $a=10^{-6}$ and $b=1$ is extremely squashed and is almost like a line in the $(R, Z)$-plane. In Fig. 14b, we have shown the associated profiles of the two principal stretches.

\section{Summary and discussion}

It is previously known that a pressurized spherical membrane balloon may bifurcate into a pear shape whereas a pressurized tubular balloon may experience localized bulging. It is shown in this paper that both types of bifurcations can occur in a pressurized ellipsoidal membrane balloon depending on its precise shape. Roughly speaking, if the balloon is of a rugby shape and is slender enough, then its behavior is more like that of a tubular balloon and will experience localized bulging. Otherwise, it may bifurcate into a pear shape. More precisely, which bifurcation will take place is determined by the ratio $b / a$ of the axes in the $Z$ - and $R$-directions, and the threshold ratio is approximately equal to 0.626 when the Ogden material model is used. We note that in the purely mechanical case under consideration, bifurcation into a pear shape is in some sense an exception since it is only permitted by the Ogden material model among the most commonly used 
material models. And even when bifurcation is possible, the bifurcated shape is hardly distinguishable from its un-bifurcated shape although the deformations are noticeably different. However, based on the recent works by ? and ?, we expect that when the balloon is also subjected to electric actuation, bifurcation into a pear shape will be the norm, and may be a major concern in the design of actuators.

We also note that the so-called localized bulging referred to in this paper is not as clean as in an infinitely long tubular balloon without imperfections. In the latter case, the tube would first experience uniform inflation until the pressure reaches a critical value, corresponding to point A in Fig.1, after which a localized bulge will appear in a dynamic fashion because of the unstable nature of the bifurcation. In reality, this "perfect" case does not exist, and imperfections always exist that will "unfold" such a bifurcation behavior. The simplest form of imperfection is the restriction imposed at the two ends when the tube is finite. This case was first studied by ? although they did not interpret their results in terms of bifurcation theory. When the tube is long enough, there exists a snap back section on the descending part of the pressure/volume diagram, but as the tube length is decreased, such a snap back section eventually disappears and we have the behavior tracing OBCDE in Fig.1. As the length is reduced even further, the bulging is no longer localized, but as Fig. 9 shows, its connection with the purely localized profile is unmistakably clear.

We have not embarked on a full-scale stability analysis although it was shown that the bifurcated configurations always have lower energy than the co-existing primary configurations. Based on the study of ?, we may expect that all the solutions on the descending branch of the pressure/volume curve will be unstable under pressure, but the bifurcated solution will be stabilized under volume or mass control when it can exist, and if it cannot exist it will be the primary solution on the descending branch that will be stabilized. These results will be verified in a separate study.

\section{References}

